\title{
Development and Validation of a Discrimination Model Between Primary PLA2R-Negative Membranous Nephropathy and Minimal Change Disease Confirmed by Renal Biopsy
}

\section{Feng Wu}

The First Affiliated Hospital of Zhengzhou University

\section{Yiding Zhang}

The First Affiliated Hospital of Zhengzhou University https://orcid.org/0000-0002-3500-1586

\section{Wen Cui}

The First Affiliated Hospital of Zhengzhou University

\section{Yijun Dong}

The First Affiliated Hospital of Zhengzhou University

\section{Yingyang Geng}

Zhengzhou University Medical School

Changhao Liu

Zhengzhou University Medical School

\section{Zemeng Li}

Zhengzhou University Medical School

\section{Yandong Xie}

Zhengzhou University Medical School

\section{Xiaojing Cai}

Zhengzhou University Medical School

Jin Shang

The First Affiliated Hospital of Zhengzhou University

Jing Xiao

The First Affiliated Hospital of Zhengzhou University

Zhanzheng Zhao ( $\square$ zhanzhengzhao@zzu.edu.cn )

The First Affiliated Hospital of Zhengzhou University https://orcid.org/0000-0001-6079-1631

\section{Research}

Keywords: Membranous nephropathy, minimal change disease, discrimination model, noninvasive identification 
Posted Date: November 19th, 2020

DOl: https://doi.org/10.21203/rs.3.rs-108746/v1

License: (c) (i) This work is licensed under a Creative Commons Attribution 4.0 International License. Read Full License 


\section{Abstract}

\section{Background}

Membranous nephropathy (MN) and minimal change disease (MCD) are two common causes leading to nephrotic syndrome (NS). They have similar clinical feature but different treatment strategy and prognosis. M-type phospholipase A2 receptor (PLA2R) is considered as a specific marker of membranous nephropathy. However, its sensitivity is only about $70 \%$. Therefore, there is a lack of effective and noninvasive tools to distinguish PLA2R-negative MN and MCD patients without renal biopsy. We aim to develop a discrimination model using noninvasive parameters for distinguishing the two diseases and support immediate treatment before result of renal biopsy.

Methods

A total 949 patients who were pathologically diagnosed as idiopathic MN or primary MCD in the First Affiliated Hospital of Zhengzhou University from January 2017 to August 2019 were enrolled in this study, including 805 idiopathic MN (605 PLAR2-positive MN and 200 PLA2R-negative MN) and 144 primary MCD. Based on the basic information and laboratory examination of 200 PLA2R-negative MN and $144 \mathrm{MCD}$, we used univariate and multivariate logistic regression analysis to select the relevant variables and develop the discrimination model. ROC curves and calibration curves were used to evaluate the diagnostic ability and calibration ability of the model. The decision curve was used to show the net benefit. We also tested the effectiveness of our model in all 949 patients.

Results

A novel model that included age, albumin, urea, high density lipoprotein, urea and red blood cell count was established for PLA2R-negative MN and MCD. The discrimination model has good differential capability (an AUC of 0.889 in training group and an AUC of 0.920 in test group) and calibration capability. When testing in all 949 patients, our model also showed good discrimination ability for all idiopathic $\mathrm{MN}$ and $\mathrm{MCD}$.

Conclusion

We constructed a discrimination model with high diagnostic effectiveness for PLA2R-negative MN and MCD. The model could also be used for all idiopathic MN and MCD patients.

\section{Introduction}

Membranous nephropathy $(\mathrm{MN})$ is one of the most common glomerular diseases causing primary nephrotic syndrome (NS) in China. The prevalence of MN has increased greatly in central China during the past two decades[1, 2]. Minimal change disease (MCD) is another primary glomerular disease with high prevalence characterized by rapid onset and development. Current studies suggest these two diseases account for about $60 \%$ NS patients in China[3, 4]. 
Renal biopsy is still the gold standard for diagnosing and distinguishing these two diseases. However, considering the poor physical condition and contraindications, many patients are unsuitable to undergo renal biopsy in daily clinical practice [5]. In addition, it does take a period of time to wait for the result. The onset of MCD is usually utterly urgent which may cause damage to renal function if the treatment is not timely and correct.

M-type phospholipase A2 receptor (PLA2R) is considered as a specific marker of idiopathic membranous nephropathy. Present evidences reveal PLA2R test has strong specificity, while its sensitivity is insufficient, 0.7 approximately[6-9]. Hence, quite amount of PLA2R-negative MN patients cannot be screened out. More importantly, the accuracy of PLA2R detection may be influenced by other diseases such as tumor-related diseases[7, 10].

Thus, it is urgent to develop a new convenient and noninvasive method to distinguish MN from MCD. The aim of our study is to develop and validate a model to distinguish MN, especially PIA2R-negative MN from MCD. The model can be used to the patients who are unsuitable or unwilling to undergo renal biopsy. We believe our model will help clinicians treat these patients in a timely manner and improve their prognosis.

\section{Methods}

\section{Study population and Ethical approval}

In this population-based retrospective analysis, we screened all the patients with NS who were hospitalized in the First Affiliated Hospital of Zhengzhou University from January 2017 to August 2019. The inclusion criteria were as follows: (1) aged 18-80 years (2) diagnosed as idiopathic MN or primary MCD by renal biopsy (3) experienced a PLA2R test. The exclusion criteria included application history of corticosteroid or immunosuppressant prior to renal biopsy. A total of 949 patients including 805 idiopathic MN (605 PLA2R-positive MN and 200 PLA2R-negative MN) and 144 primary MCD were enrolled potentially relevant cases.in this study. Among them, patients with PLA2R-negative MN and MCD were used to develop and validate the discrimination model. In the end, we also tested the differential ability of the model in all idiopathic MN and MCD. The enrollment flowchart of the participants in this study was shown in Figure1.

The First Affiliated Hospital of Zhengzhou University Ethics Review Committee granted ethical approval for the study and the ethics review approval ID was "2019-KY-361".

\section{Data collection}

We collected the basic information and laboratory examination from all patients recruited at the time of renal biopsy, which might be involved in distinguishing the two diseases. The basic information included age, gender, onset time, systolic blood pressure (SBP) and diastolic blood pressure (DBP). The laboratory indices included red blood cell (RBC) count, white blood cell (WBC) count, platelet (PLT) count, hemoglobin $(\mathrm{Hb})$ levels, mean corpuscular hemoglobin $(\mathrm{MCH})$, mean corpuscular hemoglobin concentration (MCHC), total protein (TP) levels, albumin (ALB) levels; total cholesterol (TCHO) levels, 
triglyceride $(T G)$ levels, low density lipoprotein $(L D L)$ levels, high density lipoprotein $(H D L)$ levels, estimated glomerular filtration rate (eGFR); serum creatinine (SCr) levels; uric acid (UA) levels, 24h uric total protein(24h TP) levels and 24h urine volume.

The urine and venous blood samples of all participants were collected after 12 hours of overnight fasting (except 24-hour urine samples) and sent to laboratory for testing straight away.

\section{Statistical Analysis}

The PLA2R-negetive MN and all MCD patients $(n=344)$ were randomly divided into the training group ( $n$ $=241)$ and the test group $(n=103)$.

There were a few missing data of several variables. For example, the HLD and LDL levels had 3.5\% missing values, Scr and Urea levels had $0.9 \%$ missing values. To deal with these missing data, multivariate multiple imputation with chained equations was used to impute missing values so that we could maximize the statistical power and diminish bias[11]. Descriptive statistics of all variables including means, medians, and proportions are used to describe the characteristics of two groups. The categorical data expressed as the percentages, and means \pm standard deviation (SD) or medians (quartile 1, quartile 3) were described as continuous variables satisfying or not satisfying the normal distribution, respectively. We used the univariate logistic regression to calculate the $O R$ values of the variables, selected potential variables to perform multiple logistic regression subsequently, and calculated the collinearity of the variables to remove the colinear factors. The candidate variables with a $p$ value $<0.05$ in the univariate analysis were enrolled to develop the multivariable model.

Based on the clinical features and variables with statistical sense, we attempted to develop the discrimination model for distinguishing PIA2R-negative MN from primary MCD patients.

Next, we drew a receiver operating characteristic curve (ROC) and used the area under the receiver operating characteristic curve (AUC) to evaluate the verification efficiency of the model. The calibration was assessed by constructing the calibration curve. The fitting degree of the model was assessed by the Akaike information criterion (AIC). After comprehensively evaluating the performance of each model, we obtained the best model and constructed a nomogram to make it convenient for the clinical application.

At last, we constructed the decision curve analysis to determine the clinical utility of the discrimination model by quantifying the net benefits at different threshold probabilities[12].

All the statistical analysis processes involved were completed by $R$ software, version 4.0.2. $P$ value $<0.05$ was considered statistically significant. Model validation and evaluation processes were independently performed on the training group and test group, respectively. In order to assess the diagnostic ability of the model distinguishing all idiopathic MN and MCD, we also performed validation processes on 949 potentially relevant cases. 


\section{Results}

\section{Baseline Characteristics}

The baseline characteristics of PLA2R-negative MN $(n=200)$ and MCD $(n=144)$ were shown in Table 1. The results suggested that PLA2R-negative MN patients tended to be older and have higher TP, ALB levels and greater urine volume. While, the RBC and PLT count, median Hb, TCHO, TG, LDL level, HLD, Scr, Urea, and 24hTP levels of PLA2R-negative MN were lower than those of MCD. The baseline characteristics of all idiopathic MN and MCD were shown in Table S1.

All the participants were randomly divided into training group $(n=241)$ and test group $(n=103)$. The levels of these variables were similar and had no statistical difference, which represented similar clinical profiles between the two groups (Table 2).

\section{Five potential predictors were selected to develop the discrimination model}

By means of univariate logistic regression, 14 potential predictors from 22 candidates were considered to have statistical significance $(p<0.05)$ in training group. After implementing the multivariable logistic regression analysis and removing the collinear candidates, five potential predictors including age, ALB levels, HDL levels, Urea levels and RBC counts were used to establish the discrimination model to distinguish PLA2R-negative MN from MCD (Table 3). The results indicated that patients with elder ages, higher ALB levels, lower HDL levels, lower serum Urea levels and lower RBC count were more likely suffering PLA2R-negative MN.

\section{Good discrimination and calibration capability of the model}

We drew the ROC to evaluate the diagnostic effectiveness of the model (Figure 2). The area under the ROC(AUC), which is referred to as the C-statistic, is considered to be an indicator for evaluating the effectiveness of the model. Surprisingly, we found that the discrimination model had a high efficiency with an AUC of $88.9 \%$ (cut-off value: 0.457 , sensitivity: 0.802 , specificity: 0.914 ; Figure $2 \mathrm{~A}$ ) in training group. We subsequently verified the effectiveness in the test group and the result showed an even higher efficiency with an AUC of $92.0 \%$ (cut-off value: 0.464 , sensitivity: 0.791 , specificity: 0.917 ; Figure $2 B$ ). The high value of AUC showed that the model had an good ability for discrimination the two disease.

The calibration curve was plotted to evaluate the calibration of the model and it demonstrated a good agreement between prediction and observation both in training group (mean absolute error $=0.02$ ) and test group (mean absolute error $=0.022$, Figure3 A, B). The calibration curve indicated that the model had a great calibration capability.

\section{Construction and usage of the nomogram}

In order to make the model convenient to use, we constructed the nomogram of our discrimination model based on 5 obtained predictive variables including age, HDL levels, ALB levels, Urea levels and RBC count 
(Figure 4). The value of each variable represented as a score by drawing a straight line upward from the corresponding value to the "Points" line. Sum the total points and mark it at "Total points" line. Draw down a straight line to the corresponding "MN probability" axis and obtain the possibility of MN.

\section{Decision curve showed it would add more net benefits for clinical decision}

The result of decision curve analysis for the nomogram is shown in Figure 5. The y-axis represents net benefit and the thick red line represents the model. The thin gray line represents the assumption that all patients suffer from MN, while the thin black line represents that all patients are assumed to have MCD. Both in training group or test group, the decision curve showed that if the threshold probability of a patient is $>1 \%$, using the nomogram in the present study to predict $\mathrm{MN}$ adds more benefits than performing renal biopsy on none or all patients.

\section{Diagnosis efficiency testing in potentially relevant cases}

In order to determine whether we can expand the application scope of our model, we also performed diagnosis efficiency testing in potentially relevant cases (including 605 PLA2R-positive MN, 200 PLA2Rnegative MN and $144 \mathrm{MCD}$, Figure 2). The ROC showed the good diagnostic performance with an AUC of 0.856 (cut-off value: 0.836 , sensitivity: 0.771 , specificity: 0.826 ), suggesting good discrimination ability for all idiopathic MN and MCD patients. The performances of calibration curve and decision were also good (Figure S1, S2).

\section{Discussion}

In this retrospective case-control study, we attempted to develop a discrimination model used to distinguish patients with PLA2R-negative MN and MCD. we collected and analyzed the basic information and laboratory examination of 949 patients with idiopathic MN or MCD. Based on 200 PLA2R-negative patients and $144 \mathrm{MCD}$ patients, we developed a discrimination model to differentiate the two diseases. The results showed great diagnostic effectiveness with an AUC of 0.889 in training group and an AUC of 0.920 in test group as well as high calibration capability. To the best of our knowledge, it is the first study aiming to develop a discrimination model based on the basic information and the laboratory examination of participants to distinguish primary PLA2R-negative MN and MCD. In addition, our model also showed good diagnostic effectiveness with an AUC of 0.856 in all idiopathic MN patients (either PLA2R-negative or PLA2R-positive MN) and MCD patients. It is an attempt at translational medicine of our study, which can aid clinicians to treat patients with different methods in a timely manner and thus improve their prognosis.

Currently, it is difficult to distinguish MN and MCD patients by a noninvasive tool in clinical practice. A study tried to use soluble urokinase-type plasminogen activator receptor (suPAR) level to distinguish idiopathic focal segmental glomerulosclerosis (FSGS), MN and MCD, However, the study revealed that the three types of glomerulopathy cannot be distinguished using suPAR solely [13]. Therefore, there is no miraculous indicator or model to identify these two primary glomerular diseases currently. 
Prediction and discrimination models based on clinical data have been developed increasingly in a wide variety of diseases recent years[14, 15]. In terms of kidney diseases, prediction and discrimination models are also rapidly growing owing to its scientific nature and accuracy[16-18]. The appearance of clinical models gave us great inspiration.

Present evidences suggested that MN had the largest proportion of morbidity in elderly patients, while MCD accounts for the highest proportion of primary nephrotic syndrome in young patients[3, 19, 20]. Our experiments reached similar results that the age at biopsy had a certain influence on the nomogram. In our study, patients with older age were more likely to be considered as PLA2R-negative MN, while younger age at onset was considered to be a higher risk of MCD.

ALB level was one of the predictive factors in this model. Some study showed MN patients always had higher ALB levels, which was consistent with our results[21,22]. One of the most important clinical manifestations of nephrotic syndrome is increased urinary protein and decreased albumin level. Larger amounts of glomerular albumin filtration will also make serum albumin and serum total protein at a low level. MCD patients always presents as an acute onset, severe illness, and a greater amount of urinary protein, and rapid decline in renal function may result in increased Scr levels and decreased eGFR and

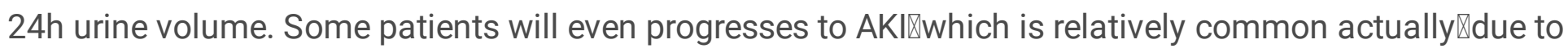
high-grade proteinuria[23]. The slit diaphragm between foot processes is regarded as a fine filter[24]. There is a common assumption that proteins leak from the slit pores due to reduced nephrin expression, leading to larger amounts of glomerular albumin filtration in MCD patients[25]. In our study, we chose ALB levels as one of the variables of the model by multivariable regression analysis to exclude the effect of collinearity.

HDL level is another variable that can be used to distinguish between two diseases according to our results. Nephrotic syndrome could cause upregulation of HDL endocytic receptor and downregulation of HDL docking receptor, causing dysregulation of lipid/lipoprotein metabolism[26]. MCD is also known as lipoid nephropathy because steatosis can be observed in epithelial cells of proximal convoluted tubules under light microscopy. In addition, increased hepatic lipoprotein synthesis and reduced lipoprotein degradation are also thought to be responsible for elevated blood lipid profiles. Takeshi Fujita compared lipid and fatty acid metabolism between $7 \mathrm{MCD}$ and $11 \mathrm{MN}$ patients. The results showed that the patients with MCD had higher level of blood lipids than MN[27]. Although the mean HDL level was much higher in patients with MCD, there was no statistical significance between the two groups, which was not exactly the same as our results. The reason might be that their sample size was not large enough, leading to the unobvious statistical significance.

Increased urea level was usually observed in a high protein decomposition status. After the renal filtration barrier disrupted, a large amount of proteins will leak into Bowman's space and renal tubules through glomerular barrier to form crude urine. When proximal tubules enhance the reabsorption of filtered proteins, the protein decomposition is also increased at the same time, resulting in elevated serum Urea level. Serum Urea level were also found different in MN patients and MCD patients in Jin Dong's 
research[28], which was consistent with our results. The Urea level plays an important role in our discrimination model.

Red blood cell count and $\mathrm{Hb}$ levels had statistical significance by means of univariate regression in our study. They are usually recognized as the indices to evaluate anemia. Compared with younger patients, idiopathic membranous nephropathy patients over 65 years old were found to have lower $\mathrm{Hb}$ level than patients less than 65 years old in Choi JY's study[29]. However, the results in Yaeni Kim's study showed there was no difference of $\mathrm{Hb}$ levels between elderly patients and young patients[30]. The reason for this ambiguity might be different gender and illness state of included patients. In our study, univariate regression showed there was no statistical difference in gender. And the data we collected was from the time of renal biopsy, reducing influence of the illness state.

The decision curve showed the clinical utility of our model, indicating it may be beneficial for clinicians to distinguish the two diseases by using our model. And using the nomogram to distinguish the two diseases added more benefits than either all or no patients who underwent a renal biopsy if the threshold probability of a patient was $>1 \%$. The results of decision curves suggest the good clinical application value of our model, reflecting the thinking mode of translational medicine.

The results of diagnosis efficiency test in potentially relevant cases suggested that our model is applicable to all idiopathic MN and MCD patients. Some hospitals are unable to perform PLA2R test, and our model might provide an alternative tool for these hospitals to distinguish MN and MCD.

Our study is an attempt in translational medicine and has a number of strengths. It had a large sample size with 949 idiopathic MN and MCD patients confirmed by renal biopsy. And the 5 items in the nomogram are routine clinical variables that can easily obtained by clinicians. We chose to collect the information and examination results at the time of renal biopsy, and excluded the influence of corticosteroid or immunosuppressive agents. What is more, our discrimination model has satisfying diagnostic effectiveness with an AUC of 0.889 in training group and an AUC of 0.920 in test group. The outstanding discrimination ability for all idiopathic MN and MCD patients even showed wider application prospects of our model. The operation of the model is simple and fast, which can help doctors diagnose patients timely. Unlike renal biopsy, our model doesn't have any contraindications so that it can be used more widely.

However, there are also several limitations in our study. First, we still need to expand the sample size for further reducing the heterogeneity. In addition, all the patients came from the First Affiliated Hospital of Zhengzhou University and we did not conduct multicenter external validation. Thus, we can't exclude the influence of diet, race and other related factors on the experimental results. Last, our model is only suitable for the identification of MN and MCD. There is still a lack of differential ability of our model for other types of nephrotic syndrome like focal segmental glomerulosclerosis (FSGS), IgA nephropathy (IgAN) and membranoproliferative glomerulonephritis (MPGN). Corrections to these shortcomings will be made in our subsequent research. 


\section{Conclusion}

In this study, we developed and validated a discrimination model used for distinguishing PLA2R-negative MN and MCD patients. We further presented a nomogram including age, ALB levels, HDL levels, urea levels and RBC counts. The model showed good discrimination and calibration ability both in training group and test group. It also had a good diagnostic performance in all MN patients and MCD patients. Hopefully, it could provide a practical and convenient tool for clinicians to distinguish these two diseases.

\section{Abbreviations}

MN: Membranous nephropathy; MCD: Minimal change disease; NS: Nephrotic syndrome; PLA2R: M-type phospholipase A2 receptor; SBP, Systolic blood pressure; DBP, Diastolic blood pressure; RBC, Red blood cell; WBC, White blood cell; PLT, Platelet; Hb, Hemoglobin; MCH, Mean corpuscular hemoglobin, MCHC: Mean corpuscular hemoglobin concentration; TP: Total protein; ALB: Albumin; TCHO, Total cholesterol; TG: Triglyceride; LDL: Low density lipoprotein; HDL: High density lipoprotein; eGFR: Estimated glomerular filtration rate; Scr: Serum creatinine; UA: Uric acid; 24hTP: 24h uric total protein; ROC: Receiver operating characteristic curve; AUC: Area under the receiver operating characteristic curve; AIC: Akaike information criterion; suPAR: Soluble urokinase-type plasminogen activator receptor; FSGS: Focal segmental glomerulosclerosis, IgAN, IgA nephropathy; MPGN: membranoproliferative glomerulonephritis.

\section{Declarations}

\section{Acknowledgements}

We would like to thank all of the patients who had contributed to this study and all the authors that participated in the research.

\section{Authors' contributions}

Zhanzheng Zhao, Xiao Jing and Jin Shang designed this study. Feng Wu and Yiding Zhang researched data and edited the manuscript. Wen Cui and Yijun Dong modified the manuscript. Yingyang Geng, Changhao Liu, Zemeng Li, Yandong Xie, and Xiaojing Cai collected the data. All authors have reviewed the manuscript. approved the final manuscript.

\section{Funding}

This work was supported by the National Natural Science Foundation of China (Grant Nos. 81873611 and 81700633), Science and Technology Innovation Team of Henan (Grant No. 17IRTSTHN020); Foundation for Leading Personnel of Central Plains of China (Grant No. 194200510006).

\section{Availability of data and materials}


All the data and materials used in our study are available from the corresponding author on reasonable request.

\section{Ethics approval and consent to participate}

The First Affiliated Hospital of Zhengzhou University Ethics Review Committee granted ethical approval for the study and the ethics review approval ID was "2019-KY-361". Informed consent was waived because of the retrospective analysis.

\section{Consent for publication}

Not applicable.

\section{Competing interests}

The authors declare that they have no competing interests

\section{Author details}

${ }^{1}$ Department of Nephrology, the First Affiliated Hospital of Zhengzhou University, Zhengzhou, China; No.1 of East Jianshe Road, Zhengzhou, Henan, P.R. China, 450052.

${ }^{2}$ Zhengzhuo University Medical School, Zhengzhou, China; No.100 of Science Avenue Zhengzhou, Henan, P.R. China, 450001

\section{References}

1. Xu, X., et al., Analysis of 4931 renal biopsy data in central China from 1994 to 2014. Renal failure, 2016. 38(7): p. 1021-30.

2. Yang, Y., et al., The Spectrum of Biopsy-Proven Glomerular Disease in China: A Systematic Review. Chinese medical journal, 2018. 131(6): p. 731-735.

3. Hu, R., et al., Spectrum of biopsy proven renal diseases in Central China: a 10-year retrospective study based on 34,630 cases. Scientific reports, 2020. 10(1): p. 10994.

4. Ng, J., et al., Causes of nephrotic syndrome and nephrotic-range proteinuria are different in adult Chinese patients: A single centre study over 33 years. Nephrology (Carlton, Vic.), 2018. 23(6): p. 565572.

5. Clinical competence in percutaneous renal biopsy. Health and Public Policy Committee. American College of Physicians. Annals of internal medicine, 1988. 108(2): p. 301-3.

6. Dai, H., H. Zhang, and Y. He, Diagnostic accuracy of PLA2R autoantibodies and glomerular staining for the differentiation of idiopathic and secondary membranous nephropathy: an updated metaanalysis. Scientific reports, 2015. 5: p. 8803. 
7. Radice, A., et al., Diagnostic specificity of autoantibodies to M-type phospholipase A2 receptor (PLA2R) in differentiating idiopathic membranous nephropathy (IMN) from secondary forms and other glomerular diseases. Journal of nephrology, 2018. 31(2): p. 271-278.

8. Du, Y., et al., The diagnosis accuracy of PLA2R-AB in the diagnosis of idiopathic membranous nephropathy: a meta-analysis. PloS one, 2014. 9(8): p. e104936.

9. Behnert, A., et al., Antiphospholipase AQ receptor autoantibodies: a comparison of three different immunoassays for the diagnosis of idiopathic membranous nephropathy. Journal of immunology research, 2014. 2014: p. 143274.

10. Zhang, D., et al., Clinicopathological features in membranous nephropathy with cancer: $A$ retrospective single-center study and literature review. The International journal of biological markers, 2019. 34(4): p. 406-413.

11. Buuren, S., Groothuis-Oudshoorn K.mice: Multivariate Imputation by Chained Equations in R. J Statistical Software, 2011. 45.

12. Vickers, A.J., et al., Extensions to decision curve analysis, a novel method for evaluating diagnostic tests, prediction models and molecular markers. BMC Medical Informatics and Decision Making, 2008. 8(1): p. 53.

13. Segarra, A., et al., [Diagnostic value of soluble urokinase-type plasminogen activator receptor serum levels in adults with idiopathic nephrotic syndrome]. Nefrologia : publicacion oficial de la Sociedad Espanola Nefrologia, 2014. 34(1): p. 46-52.

14. Ghadri, J., et al., A novel clinical score (InterTAK Diagnostic Score) to differentiate takotsubo syndrome from acute coronary syndrome: results from the International Takotsubo Registry. European journal of heart failure, 2017. 19(8): p. 1036-1042.

15. Kamada, Y., et al., A novel noninvasive diagnostic method for nonalcoholic steatohepatitis using two glycobiomarkers. Hepatology (Baltimore, Md.), 2015. 62(5): p. 1433-43.

16. Jiang, S., et al., Novel Model Predicts Diabetic Nephropathy in Type 2 Diabetes. American journal of nephrology, 2020. 51(2): p. 130-138.

17. Sun, L., et al., Development and validation of a predictive model for end-stage renal disease risk in patients with diabetic nephropathy confirmed by renal biopsy. PeerJ, 2020. 8: p. e8499.

18. Cheng, Y., et al., Development and validation of a predictive model for the progression of diabetic kidney disease to kidney failure. Renal failure, 2020. 42(1): p. 550-559.

19. Yim, T., et al., Patterns in renal diseases diagnosed by kidney biopsy: A single-center experience. Kidney research and clinical practice, 2020. 39(1): p. 60-69.

20. Zhou, Q., et al., Changes in the diagnosis of glomerular diseases in east China: a 15-year renal biopsy study. Renal failure, 2018. 40(1): p. 657-664.

21. Zhang, Q., et al., Antiphospholipid Antibodies in Patients with Membranous Nephropathy. Nephron, 2019. 143(4): p. 228-233. 
22. Yamamoto, R., et al., Incidence of remission and relapse of proteinuria, end-stage kidney disease, mortality, and major outcomes in primary nephrotic syndrome: the Japan Nephrotic Syndrome Cohort Study (JNSCS). Clinical and experimental nephrology, 2020. 24(6): p. 526-540.

23. Waldman, M., et al., Adult minimal-change disease: clinical characteristics, treatment, and outcomes. Clinical journal of the American Society of Nephrology : CJASN, 2007. 2(3): p. 445-53.

24. Deen, W., M. Lazzara, and B. Myers, Structural determinants of glomerular permeability. American journal of physiology. Renal physiology, 2001. 281(4): p. F579-96.

25. Lahdenkari, A., et al., Podocytes are firmly attached to glomerular basement membrane in kidneys with heavy proteinuria. Journal of the American Society of Nephrology : JASN, 2004. 15(10): p. 26118.

26. Vaziri, N., et al., Nephrotic syndrome causes upregulation of HDL endocytic receptor and PDZK-1dependent downregulation of HDL docking receptor. Nephrology, dialysis, transplantation : official publication of the European Dialysis and Transplant Association - European Renal Association, 2011. 26(10): p. 3118-23.

27. Fujita, T., et al., Comparison of lipid and fatty acid metabolism between minimal change nephrotic syndrome and membranous nephropathy. In vivo (Athens, Greece), 2006. 20: p. 891-3.

28. Dong, J., et al., A pilot and comparative study between pathological and serological levels of immunoglobulin and complement among three kinds of primary glomerulonephritis. BMC immunology, 2018. 19(1): p. 18.

29. Choi, J., et al., Idiopathic membranous nephropathy in older patients: Clinical features and outcomes. PloS one, 2020. 15(10): p. e0240566.

30. Kim, Y., et al., Clinical outcomes and effects of treatment in older patients with idiopathic membranous nephropathy. The Korean journal of internal medicine, 2019. 34(5): p. 1091-1099.

\section{Tables}

Table 1. Baseline characteristics of PLA2R-negative MN and MCD. SBP, systolic blood pressure; DBP, diastolic blood pressure; RBC, red blood cell; WBC, white blood cell; PLT, platelet; $\mathrm{Hb}$, hemoglobin; $\mathrm{MCH}$, mean corpuscular hemoglobin, MCHC: mean corpuscular hemoglobin concentration; TP: total protein; ALB: albumin; TCHO, total cholesterol; TG: triglyceride; LDL: low density lipoprotein; HDL: high density lipoprotein; eGFR: estimated glomerular filtration rate; Scr: serum creatinine; UA: uric acid; 24hTP: 24h uric total protein. 


\begin{tabular}{|llll|}
\hline Variables & MN $(\mathbf{n}=\mathbf{2 0 0})$ & $\mathrm{MCD}(\mathbf{n}=144)$ & $\mathbf{p}$ Value \\
\hline Age & $50(34,58)$ & $31(24,48)$ & $<0.001$ \\
\hline Gender, n (male, \%) & $114(57.0)$ & $80(55.6)$ & 0.790 \\
\hline SBP & $130(120,140)$ & $127(117,135)$ & 0.114 \\
\hline DBP & $80(76,87)$ & $83(78,90)$ & 0.117 \\
\hline RBC & $4.33 \pm 0.58$ & $4.64 \pm 0.64$ & $<0.001$ \\
\hline WBC & $6.50(4.50,7.88)$ & $6.2(5.1,7.6)$ & 0.310 \\
\hline PLT & $225(192,265)$ & $252(203,313)$ & 0.001 \\
\hline Hb & $130.5 \pm 18.1$ & $138.9 \pm 21.9$ & $<0.001$ \\
\hline MCH & $30.3(29.3,31.4)$ & $30.4(29.0,31.4)$ & 0.878 \\
\hline MCHC & $333(327,339)$ & $333(326,338)$ & 0.515 \\
\hline TP & $51.0(44.7,58.1)$ & $41.2(36.4,46.8)$ & $<0.001$ \\
\hline ALB & $28.05(22.81,34.38)$ & $19.78(16.83,23.18)$ & $<0.001$ \\
\hline TCHO & $6.05(4.95,7.83)$ & $10.06(7.86,11.93)$ & $<0.001$ \\
\hline TG & $1.85(1.25,2.67)$ & $2.18(1.51,3.20)$ & 0.018 \\
\hline LDL & $3.99(3.11,5.33)$ & $7.82(5.34,9.66)$ & $<0.001$ \\
\hline HDL & $1.25(1.05,1.57)$ & $1.63(1.29,2.05)$ & $<0.001$ \\
\hline eGFR & $104.03(90.24,114.49)$ & $100.16(71.38,118.85)$ & 0.383 \\
\hline Scr & $68(56,80)$ & $76(61,102)$ & $<0.001$ \\
\hline Urea & $4.80(3.73,6.02)$ & $5.50(3.99,8.38)$ & 0.001 \\
\hline UA & $310.0(259.3,375.3)$ & $317.5(261.5,385.3)$ & 0.650 \\
\hline 24hTP & $6.04(1.92,7.20)$ & $6.43(4.28,9.04)$ & $<0.001$ \\
\hline Urine volume & $1.6(1.2,2.2)$ & $1.2(0.7,1.9)$ & $<0.001$ \\
\hline
\end{tabular}

Table 2. Baseline characteristics showed there was no statistical difference in the training group and test group. SBP, systolic blood pressure; DBP, diastolic blood pressure; RBC, red blood cell; WBC, white blood cell; PLT, platelet; $\mathrm{Hb}$, hemoglobin; $\mathrm{MCH}$, mean corpuscular hemoglobin, $\mathrm{MCHC}$ : mean corpuscular hemoglobin concentration; TP: total protein; ALB: albumin; TCHO, total cholesterol; TG: triglyceride; LDL: low density lipoprotein; HDL: high density lipoprotein; eGFR: estimated glomerular filtration rate; Scr: serum creatinine; UA: uric acid; 24hTP: $24 \mathrm{~h}$ uric total protein. 


\begin{tabular}{|llll|}
\hline Variables & Training group(n=241) & Validation group(n=103) & p Value \\
\hline Age & $40(29,54)$ & $48(31,55)$ & 0.112 \\
\hline Gender, $n$ (male, \%) & $131(54.3)$ & $63(61.1)$ & 0.244 \\
\hline SBP & $130(119,139)$ & $128(120,139)$ & 0.745 \\
\hline DBP & $81(77,90)$ & $80(76,86)$ & 0.278 \\
\hline RBC & $4.47 \pm 0.63$ & $4.52 \pm 0.61$ & 0.706 \\
\hline WBC & $6.50(5.30,7.90)$ & $6.1(5.2,7.6)$ & 0.268 \\
\hline PLT & $234(197,279)$ & $236(186,286)$ & 0.694 \\
\hline Hb & $134.4 \pm 20.6$ & $133.3 \pm 19.3$ & 0.648 \\
\hline MCH & $30.4(29.3,31.5)$ & $30.2(29.0,31.4)$ & 0.863 \\
\hline MCHC & $333(327,340)$ & $333(327,337)$ & 0.459 \\
\hline TP & $46.6(40.6,54.4)$ & $45.8(39.7,53.0)$ & 0.648 \\
\hline ALB & $24.0(19.5,30.8)$ & $22.9(18.5,31.0)$ & 0.439 \\
\hline TCHO & $7.79(5.70,10.29)$ & $6.90(5.09,9.64)$ & 0.095 \\
\hline TG & $2.08(1.36,2.94)$ & $1.85(1.42,2.50)$ & 0.219 \\
\hline LDL & $5.14(3.65,7.88)$ & $4.89(3.31,8.19)$ & 0.472 \\
\hline HDL & $1.41(1.12,1.79)$ & $1.32(1.09,1.78)$ & 0.490 \\
\hline eGFR & $105.36(88.83,117.63)$ & $98.96(80.73,110.67)$ & 0.057 \\
\hline Scr & $69.0(58.5,84.5)$ & $73.0(60.0,90.9)$ & 0.141 \\
\hline Urea & $4.90(3.80,6.75)$ & $5.00(4.00,6.60)$ & 0.696 \\
\hline UA & $309(254,377)$ & $324.0(273,395)$ & 0.094 \\
\hline $24 h T P$ & $5.16(2.55,7.81)$ & 0.971 \\
\hline Urine volume & $1.5(1.0,2.1)$ & & 0.787 \\
\hline
\end{tabular}

Table 3. Potential risk factors identified by univariate and multivariate logistic regression analysis. SBP, systolic blood pressure; DBP, diastolic blood pressure; RBC, red blood cell; WBC, white blood cell; PLT, platelet; $\mathrm{Hb}$, hemoglobin; $\mathrm{MCH}$, mean corpuscular hemoglobin, $\mathrm{MCHC}$ : mean corpuscular hemoglobin concentration; TP: total protein; ALB: albumin; TCHO, total cholesterol; TG: triglyceride; LDL: Iow density lipoprotein; HDL: high density lipoprotein; eGFR: estimated glomerular filtration rate; Scr: serum creatinine; UA: uric acid; 24hTP: $24 \mathrm{~h}$ uric total protein. 


\begin{tabular}{|lllll|}
\hline Variable & Univariable & & Multivariable & \\
\hline & OR (95\%Cl) & P & OR (95\%Cl) & P \\
\hline Age & $1.053(1.033-1.073)$ & $<0.001$ & $1.057(1.024-1.090)$ & $<0.001$ \\
\hline Gender & $0.927(0.555-1.550)$ & 0.773 & & \\
\hline SBP & $1.016(1.000-1.032)$ & 0.045 & & \\
\hline DBP & $0.987(0.963-1.011)$ & 0.288 & & \\
\hline RBC & $0.385(0.243-0.611)$ & $<0.001$ & $0.313(0.102-0.959)$ & 0.042 \\
\hline WBC & $1.062(0.932-1.210)$ & 0.365 & & \\
\hline PLT & $0.993(0.989-0.997)$ & 0.001 & & \\
\hline Hb & $0.978(0.965-0.991)$ & 0.001 & & \\
\hline MCH & $1.036(0.914-1.174)$ & 0.582 & & \\
\hline MCHC & $1.012(0.988-1.038)$ & 0.329 & & \\
\hline TP & $1.116(1.077-1.157)$ & $<0.001$ & & \\
\hline ALB & $1.174(1.118-1.234)$ & $<0.001$ & $1.190(1.044-1.357)$ & 0.009 \\
\hline TCHO & $0.656(0.582-0.740)$ & $<0.001$ & & \\
\hline TG & $1.010(0.893-1.142)$ & 0.876 & & \\
\hline LDL & $0.647(0.570-0.734)$ & $<0.001$ & & \\
\hline HDL & $0.251(0.142-0.445)$ & $<0.001$ & $0.258(0.121-0.549)$ & $<001$ \\
\hline eGFR & $1.007(0.998-1.017)$ & 0.122 & & \\
\hline Scr & $0.992(0.986-0.999)$ & 0.016 & & \\
\hline Urea & $0.851(0.775-0.934)$ & 0.001 & $0.822(0.684-0.988)$ & \\
\hline UA & $0.999(0.996-1.022)$ & 0.500 & & \\
\hline 24hTP & $0.865(0.803-0.930)$ & $<0.001$ & & \\
\hline Urine volume & $1.945(1.354-2.794)$ & $<0.001$ & & \\
\hline & & & & \\
\hline
\end{tabular}

\section{Figures}


Figure 1

nclusion criteria:

1. Aged $18-80$ years

2. Pathologically diagnosed as primary $M C D$ or primary $M N$

3. Experienced a PLA2R test $(n=1175, M C D=184, M N=991)$

Testing the diagnosis
efficiency of the
disceimination model
in potentially relevant
cases

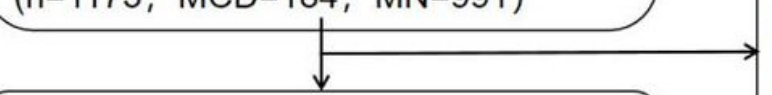

Corticosteroid or immunosuppressive therapy before renal biopsy $(n=226, M C D=40, M N=186)$

Excluded:

Positive result of PLA2R $(n=605, M C D=0, M N=605)$

Cases used to develop and validate the discrimination model $(n=344, M C D=144, M N=200)$

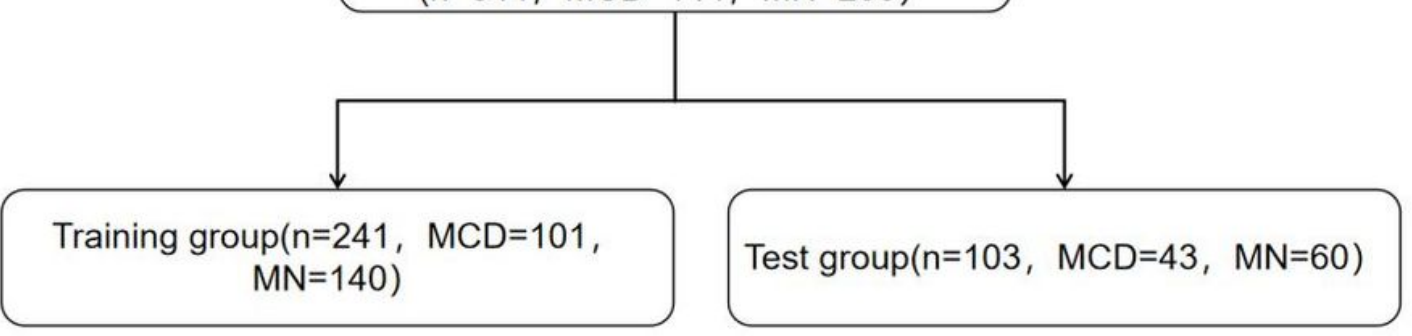

\section{Figure 1}

Enrollment flowchart of participants used for model development and validation. MCD, Minimal change disease; MN, Membranous nephropathy.

Figure 1

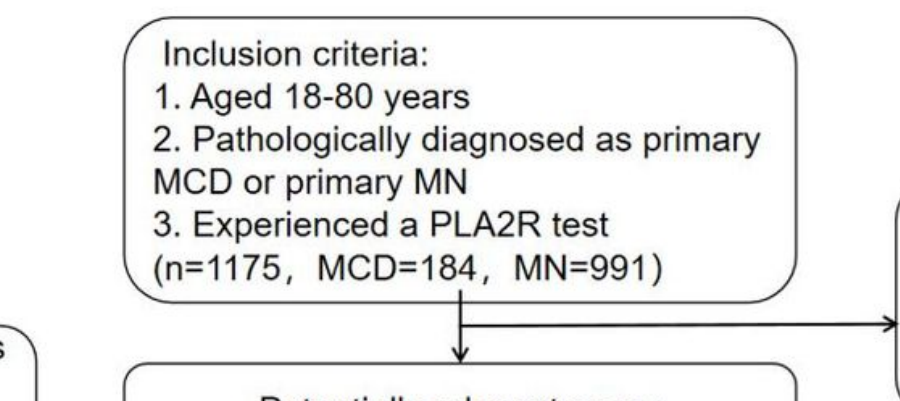

Excluded:

Corticosteroid or immunosuppressive therapy before renal biopsy

Testing the diagnosis
efficiency of the
disceimination model
in potentially relevant
cases

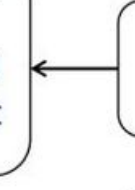

Potentially relevant cases $(n=226, M C D=40, M N=186)$ $(n=949, M C D=144, M N=805)$

Excluded:

Positive result of PLA2R $(n=605, M C D=0, M N=605)$

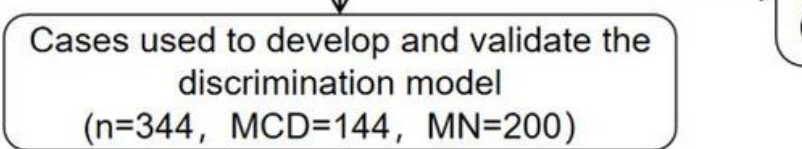

$$
\begin{aligned}
& \text { discrimination model } \\
& 44, M C D=144, M N=200 \text { ) }
\end{aligned}
$$

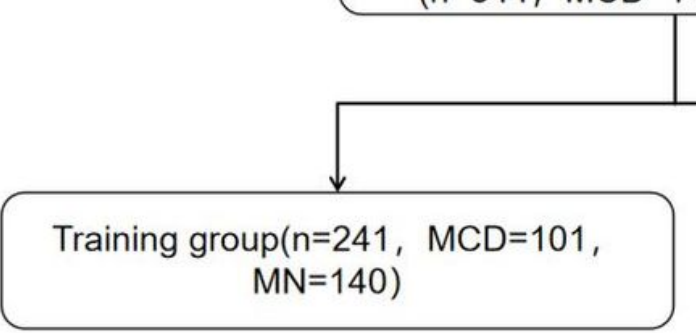

Test group $(n=103, M C D=43, M N=60)$ 


\section{Figure 1}

Enrollment flowchart of participants used for model development and validation. MCD, Minimal change disease; MN, Membranous nephropathy.

Figure 2

A

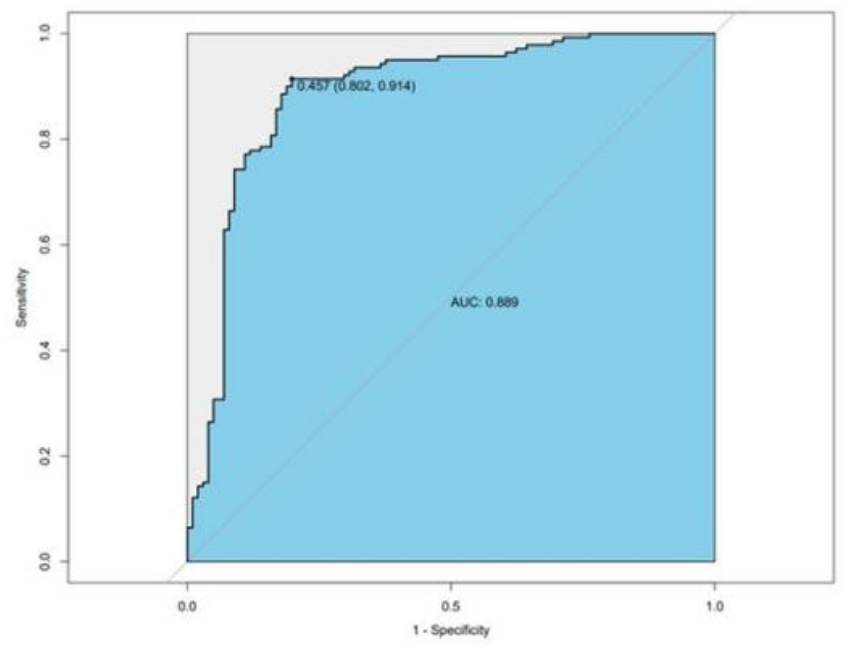

B

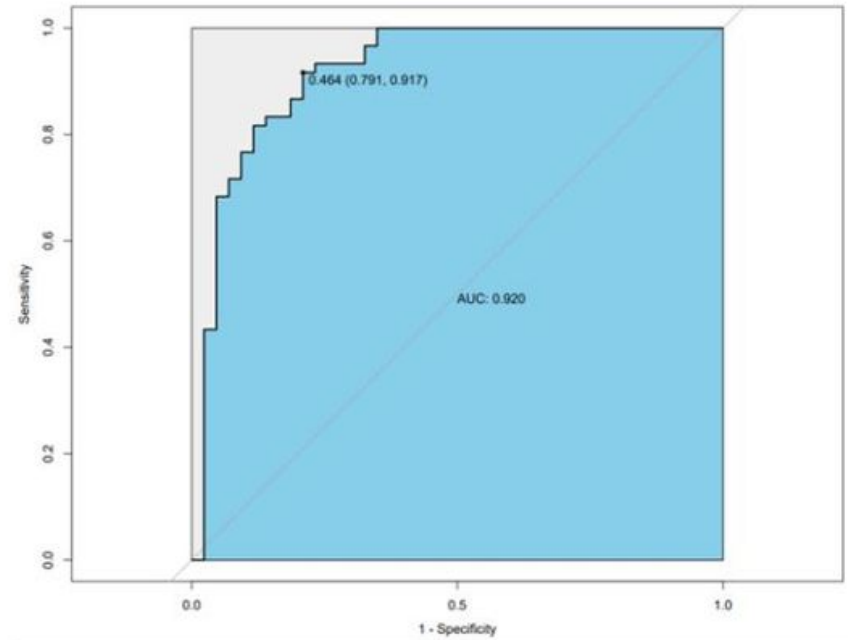

\section{Figure 2}

Differential capability of the nomogram. (A)ROC curve based on obtained potential risk factors identified by multivariate logistic regression analysis showing discrimination rate for PLA2R negative MN and MCD in the training group. (B)ROC curve based on obtained potential risk factors identified by multivariate logistic regression analysis showing discrimination rate for PLA2R negative $M N$ and $M C D$ in the test group. MCD, Minimal change disease; MN, Membranous nephropathy.

Figure 2

A

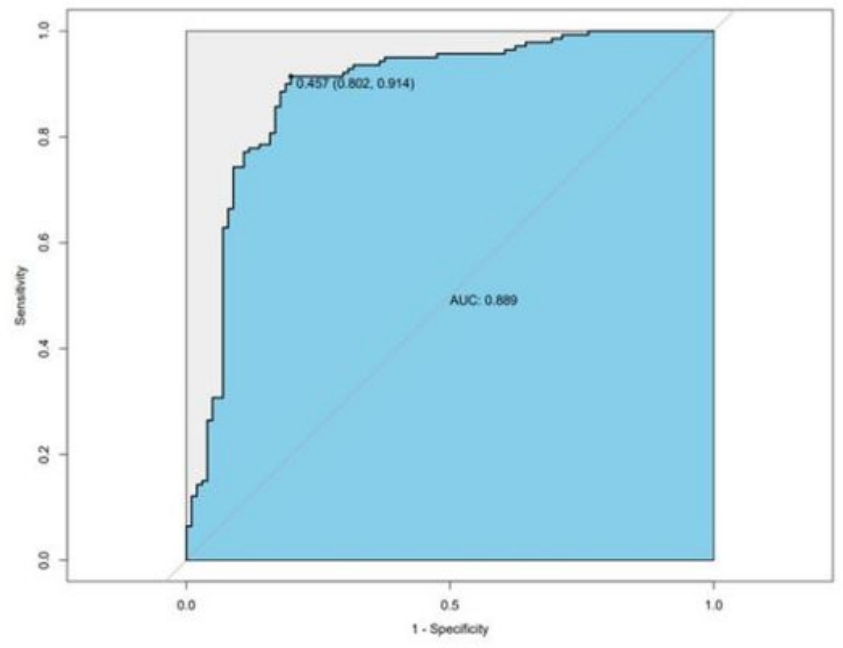

B

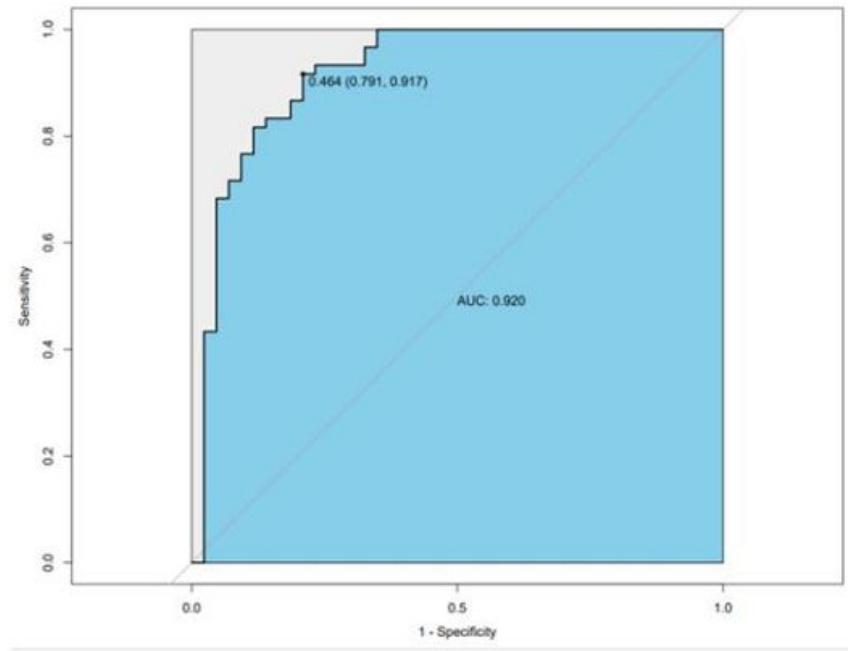


Figure 2

Differential capability of the nomogram. (A)ROC curve based on obtained potential risk factors identified by multivariate logistic regression analysis showing discrimination rate for PLA2R negative MN and MCD in the training group. (B)ROC curve based on obtained potential risk factors identified by multivariate logistic regression analysis showing discrimination rate for PLA2R negative $M N$ and $M C D$ in the test group. MCD, Minimal change disease; MN, Membranous nephropathy.

Figure 3

A

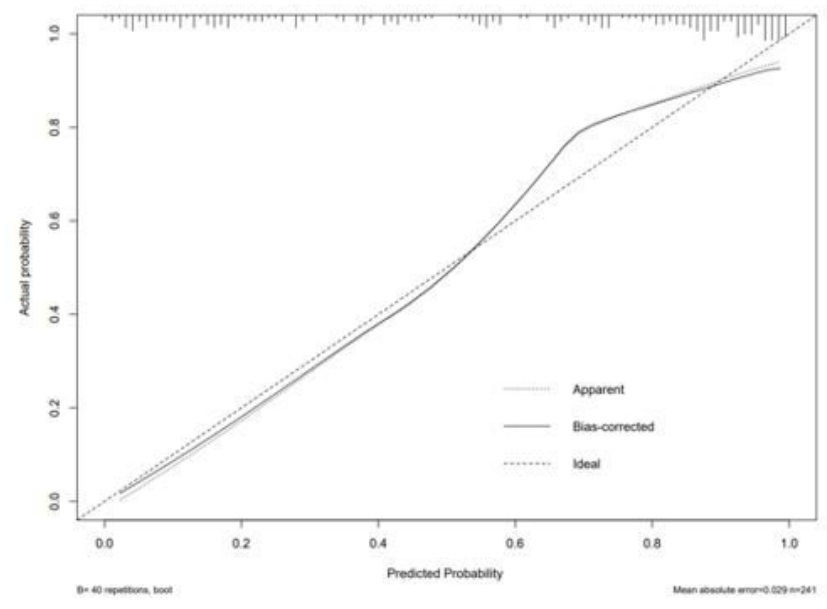

B

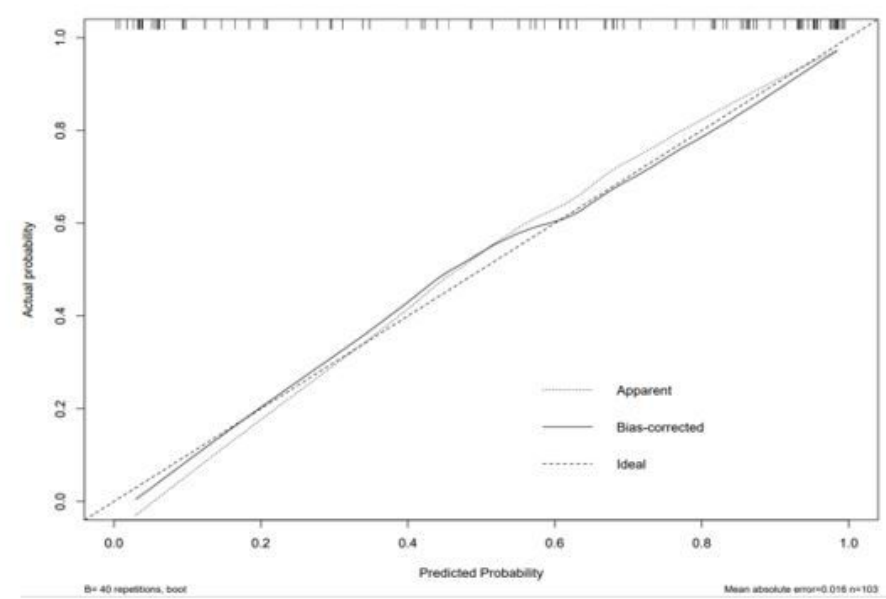

Figure 3

Calibration curve of the discrimination nomogram in the (A)training group or (B)test group. The $x$-axis represents the predicted probability of $\mathrm{MN}$. The y-axis represents the actual pathologically diagnosed $\mathrm{MN}$. The diagonal dotted line represents a perfect prediction by an ideal model. The solid line represents the performance of the nomogram, of which a closer fit to the diagonal dotted line represents a better prediction. MCD, Minimal change disease; MN, Membranous nephropathy. 
Figure 3

A

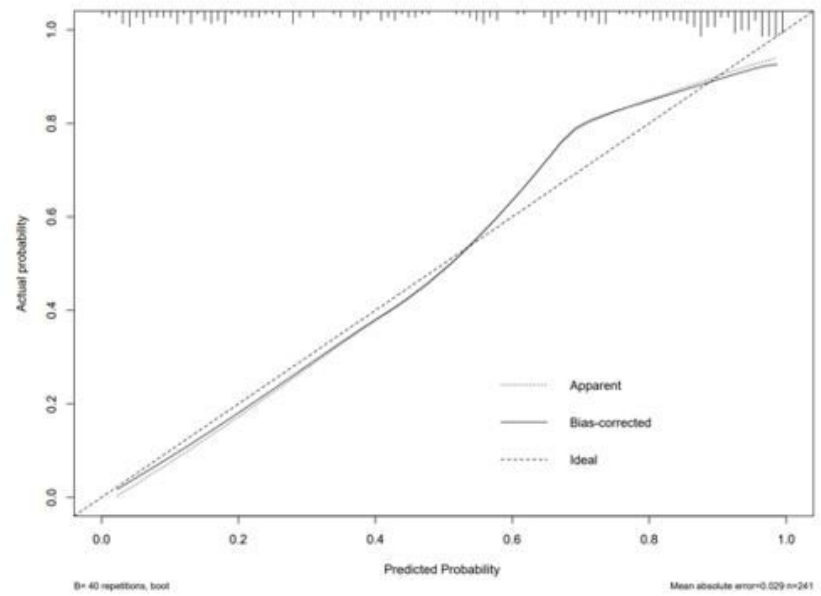

B

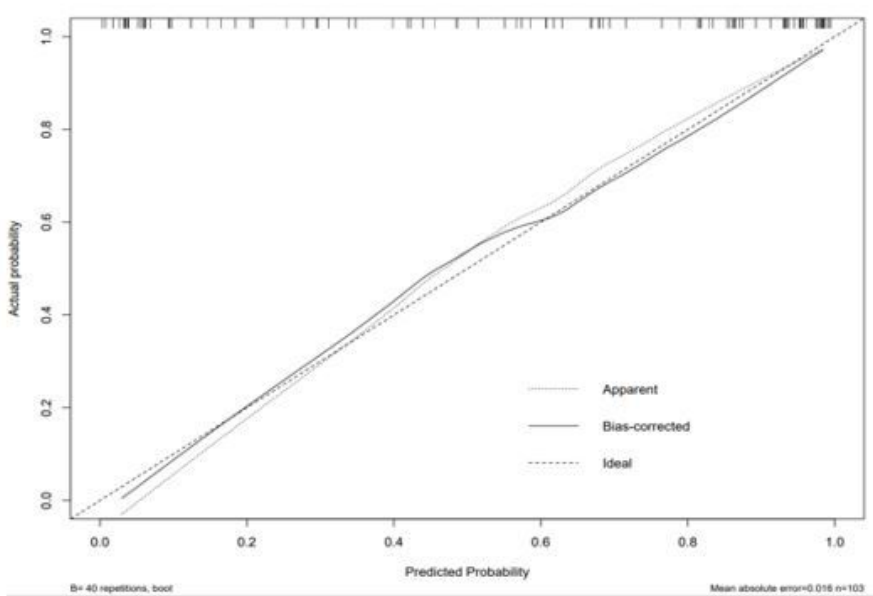

\section{Figure 3}

Calibration curve of the discrimination nomogram in the (A)training group or (B)test group. The $x$-axis represents the predicted probability of $\mathrm{MN}$. The $\mathrm{y}$-axis represents the actual pathologically diagnosed $\mathrm{MN}$. The diagonal dotted line represents a perfect prediction by an ideal model. The solid line represents the performance of the nomogram, of which a closer fit to the diagonal dotted line represents a better prediction. MCD, Minimal change disease; MN, Membranous nephropathy. 
Figure 4

$$
\text { Points }
$$

$$
0
$$

10

20

30

40

50

60

$70 \quad 80$

$90 \quad 100$
+

Age

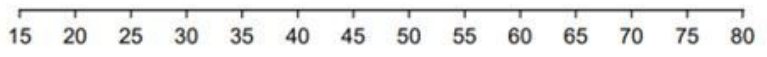

ALB

\begin{tabular}{rllllllll}
\hline 10 & 15 & 20 & 25 & 30 & 35 & 40 & 45 & 50
\end{tabular}

HDL

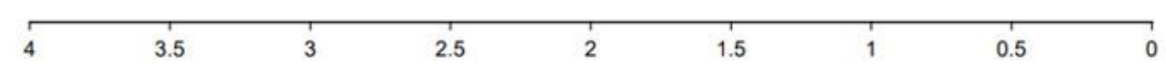

Urea

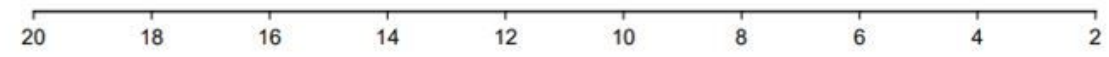

RBC

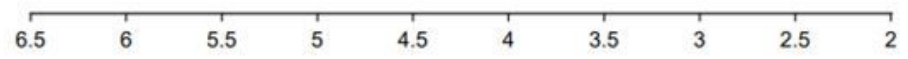

Total Points

\begin{tabular}{ll|l|l|l|l|l|l|}
\hline & 100 & 150 & 200 & 250 & 300 & 350
\end{tabular}

MN Probability

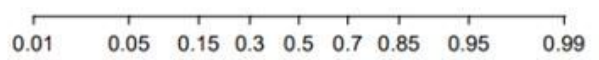

\section{Figure 4}

Nomogram based on the laboratory model; ALB, Albumin; HDL, High density lipoprotein; RBC, Red blood cell. MN, Membranous nephropathy. 
Figure 4

Points

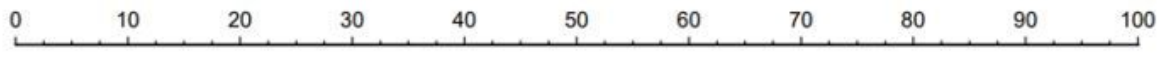

Age

$\begin{array}{llllllllllllll}15 & 20 & 25 & 30 & 35 & 40 & 45 & 50 & 55 & 60 & 65 & 70 & 75 & 80\end{array}$

ALB

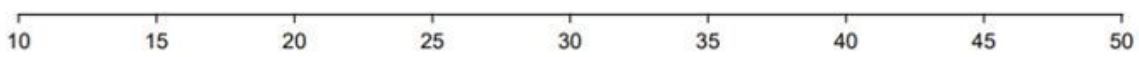

HDL

\begin{tabular}{lllllllll}
\hline 4 & 3.5 & 3 & 2.5 & 2 & 1.5 & 1 & 0.5 & 0
\end{tabular}

Urea

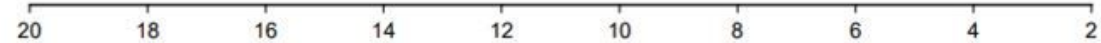

RBC

$\begin{array}{llllllllll}6.5 & 6 & 5.5 & 5 & 4.5 & 4 & 3.5 & 3 & 2.5 & 2\end{array}$

Total Points

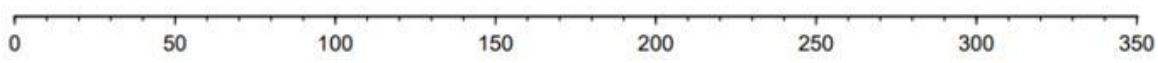

MN Probability

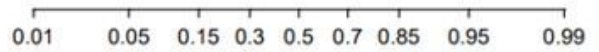

\section{Figure 4}

Nomogram based on the laboratory model; ALB, Albumin; HDL, High density lipoprotein; RBC, Red blood cell. MN, Membranous nephropathy.

Figure 5

A

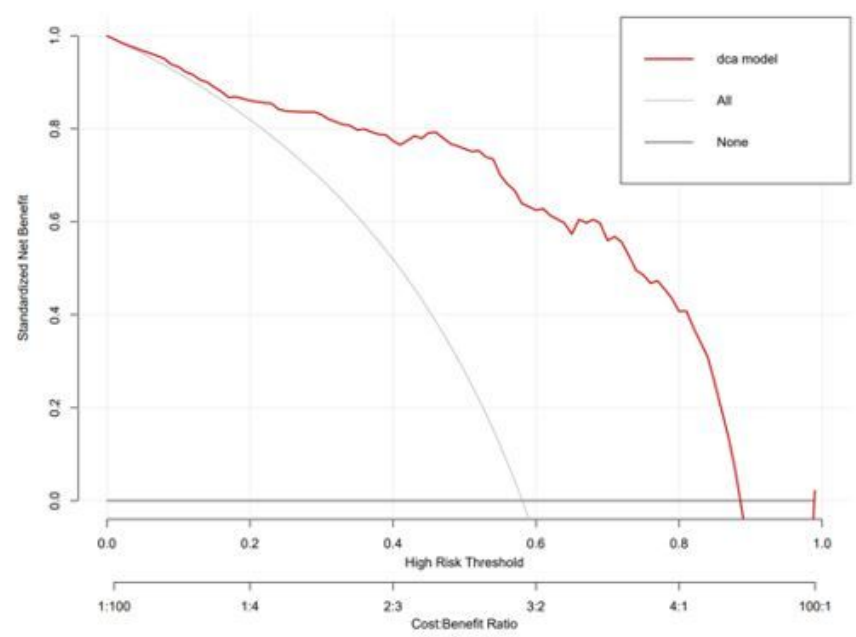

B

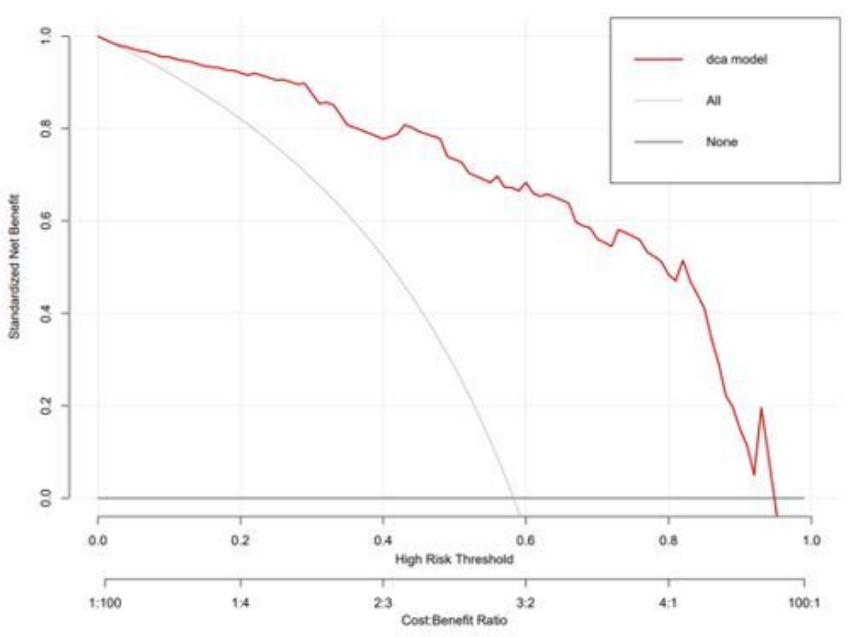




\section{Figure 5}

Decision curve for the nomogram predicting $\mathrm{MN}$ in (A)training group or (B)test group. Net benefit is shown on the y-axis. The thick red line represents the model; the thin gray line represents the assumption that all patients have $\mathrm{MN}$; the thin black line represents the assumption that all patients have MCD. Both in training group or test group, the decision curve showed that if the threshold probability of a patient is > $1 \%$, using the nomogram in the present study to predict $\mathrm{MN}$ adds more benefit than performing biopsy on all or no patients. MCD, Minimal change disease; MN, Membranous nephropathy.

Figure 5

A

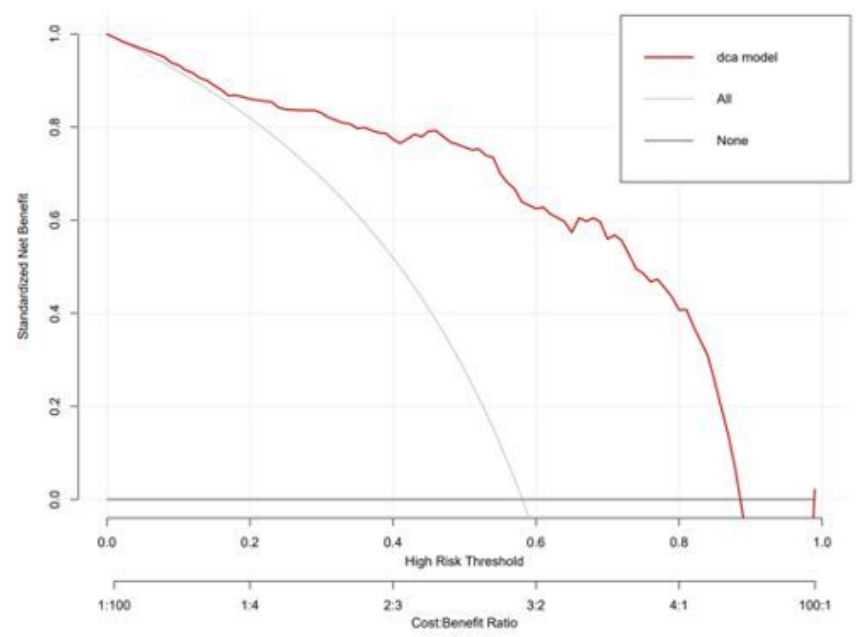

B

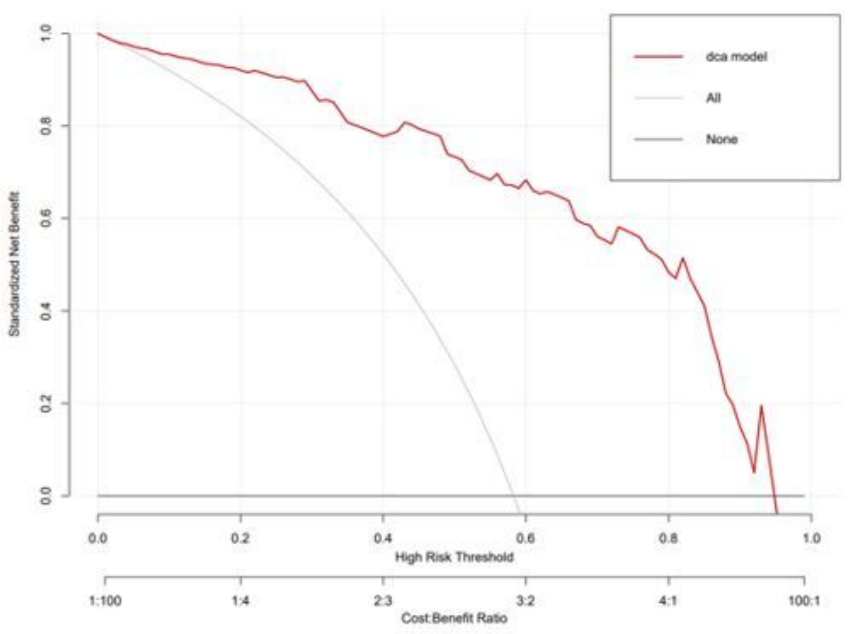

\section{Figure 5}

Decision curve for the nomogram predicting $\mathrm{MN}$ in (A)training group or (B)test group. Net benefit is shown on the $y$-axis. The thick red line represents the model; the thin gray line represents the assumption that all patients have $\mathrm{MN}$; the thin black line represents the assumption that all patients have MCD. Both in training group or test group, the decision curve showed that if the threshold probability of a patient is > $1 \%$, using the nomogram in the present study to predict $\mathrm{MN}$ adds more benefit than performing biopsy on all or no patients. MCD, Minimal change disease; MN, Membranous nephropathy. 
Figure 6

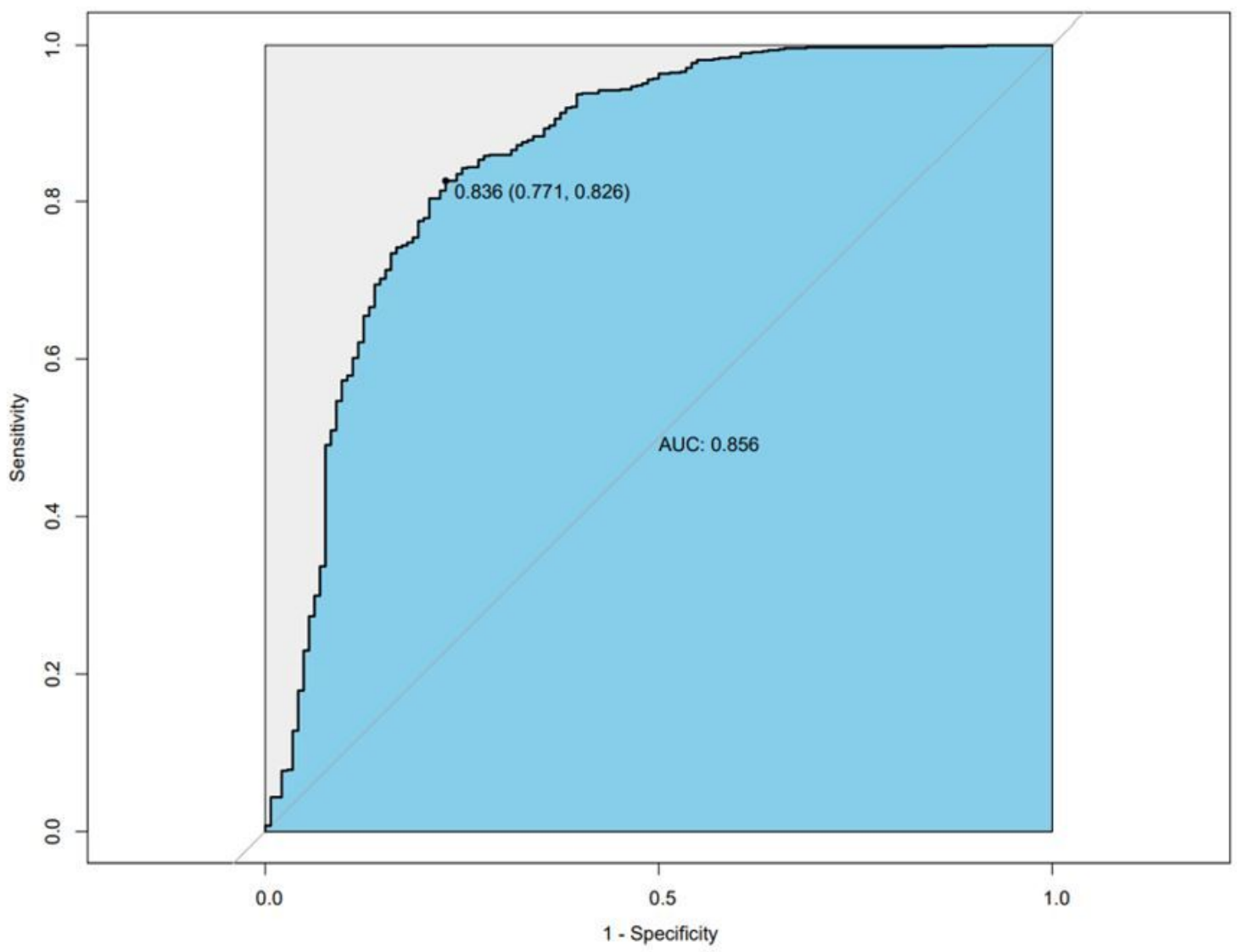

Figure 6

ROC curve showing discrimination effective of nomogram used in all MN and MCD. MCD, Minimal change disease; MN, Membranous nephropathy. 
Figure 6

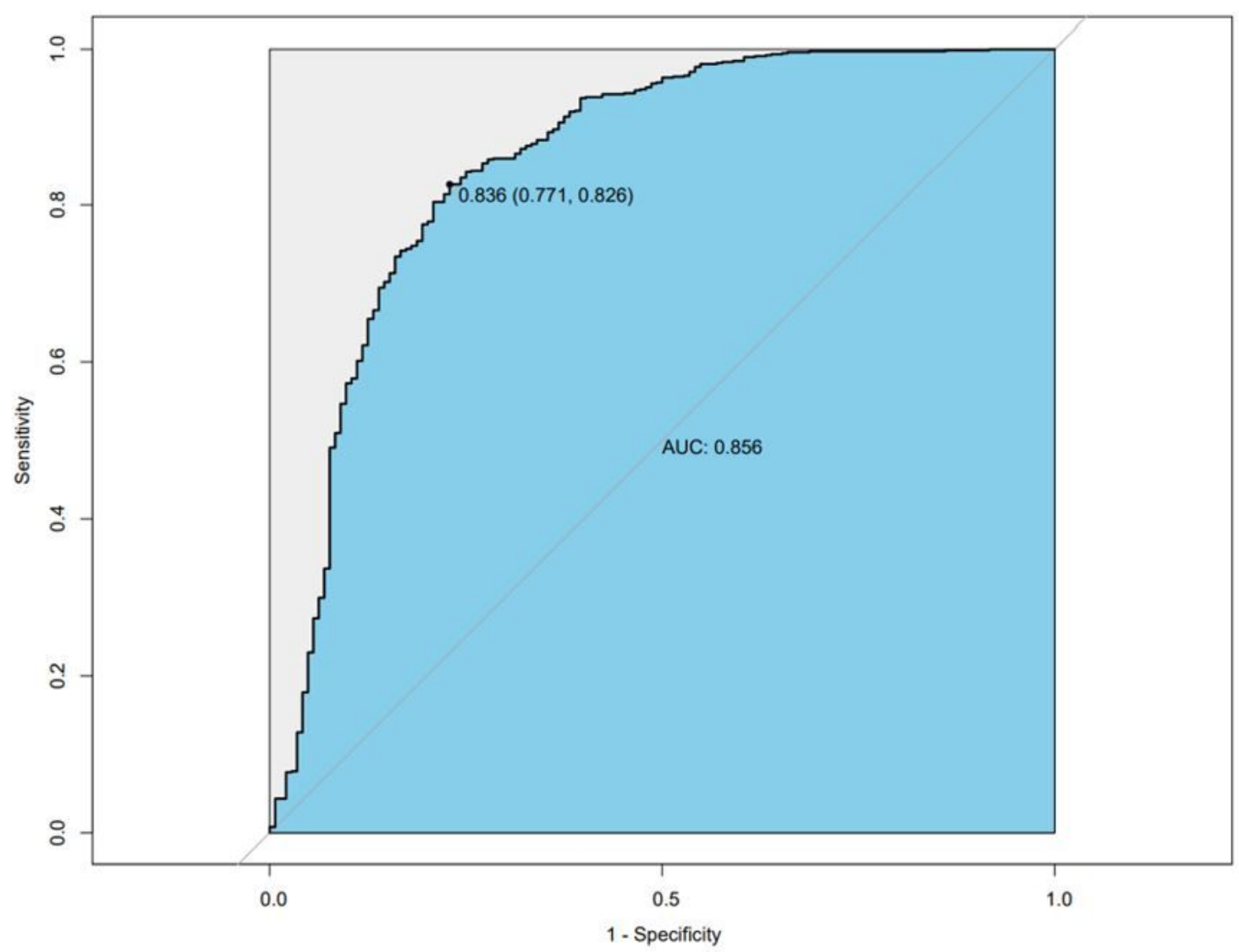

Figure 6

ROC curve showing discrimination effective of nomogram used in all MN and MCD. MCD, Minimal change disease; MN, Membranous nephropathy.

\section{Supplementary Files}

This is a list of supplementary files associated with this preprint. Click to download.

- FigureS1.pdf

- Figures1.pdf

- Figures2.pdf

- FigureS2.pdf

- Tables1.docx

- Tables1.docx 